\title{
MicroRNA-Biogenesis and Pre-mRNA Splicing Crosstalk
}

\author{
Noam Shomron ${ }^{1}$ and Carmit Levy ${ }^{2}$ \\ ${ }^{1}$ Department of Cell and Developmental Biology, Sackler Faculty of Medicine, Tel Aviv University, 69978 Tel Aviv, Israel \\ ${ }^{2}$ Massachusetts General Hospital, Harvard Medical School, Charlestown, MA 02129, USA
}

Correspondence should be addressed to Noam Shomron, nshomron@post.tau.ac.il

Received 27 March 2009; Accepted 18 May 2009

Recommended by Zhumur Ghosh

MicroRNAs (miRNAs) are often hosted in introns of protein-coding genes. Given that the same transcriptional unit can potentially give rise to both miRNA and mRNA transcripts raises the intriguing question of the level of interaction between these processes. Recent studies from transcription, pre-mRNA splicing, and miRNA-processing perspectives have investigated these relationships and yielded interesting, yet somewhat controversial findings. Here we discuss major studies in the field.

Copyright () 2009 N. Shomron and C. Levy. This is an open access article distributed under the Creative Commons Attribution License, which permits unrestricted use, distribution, and reproduction in any medium, provided the original work is properly cited.

\section{Transcription, Pre-mRNA Splicing, and miRNA Biogenesis}

The gene expression pathway initiates at nuclear transcription generating a pre-mRNA, which very often undergoes splicing, post-transcriptional regulation, and then translation into a protein. During the pre-mRNA splicing process, introns are removed and exons are joined in order to generate the mature mRNA [1-3]. The highly coordinated splicing event takes place in a large complex called the Spliceosome. The formation of this functional megacomplex is an orchestrated assembly of proteins and RNA that requires identification of exon-intron boundaries [4]. Exons are regularly alternatively spliced, meaning that they are either included or excluded from the final mature mRNA transcript. A recent comprehensive sequencing study observed that more than $90 \%$ of the genes undergo alternative splicing [5]. This vastly increases the transcriptome repertoire, and emphasizes both the significance of splicing and the requirement for its accurate execution.

In addition to protein coding genes, noncoding genes are transcribed. microRNAs (miRNA), the most comprehensive noncoding group, are a class of $\sim 22 \mathrm{nt}$ noncoding RNAs that inhibit gene expression through binding to the $3^{\prime}$ UnTranslated Region (UTR) of target mRNA transcripts $[6,7]$. There are hundreds of unique miRNAs in a given species [8], each predicted to regulate a plethora of target genes [9-13]. In fact, computational predictions indicate that
miRNAs may regulate $60 \%$ of all human protein coding genes [14]. Therefore, it came as no surprise that miRNAs were linked to many cellular processes such as differentiation, growth, and apoptosis [15], while miRNA perturbations were associated with numerous diseases, including cancer $[16,17]$. In the past few years, the pivotal role played by miRNAs in gene regulation has been recognized [1820].

miRNAs are processed through a series of posttranscriptional biogenesis steps. The canonical maturation pathway, similar to protein-coding genes, initiates at transcription (mostly by RNA polymerase II) generating a primary (pri-) miRNA. The pri-miRNA is characterized by a hairpin RNA structure recognized by the nuclear RNAseIII enzyme Drosha, and its cofactor DGCR8 [21]. These proteins work in a complex of several proteins, known as the Microprocessor. The Microprocessor cleaves the primiRNA to generate a shorter hairpin of about $70 \mathrm{nt}$ lengththe pre-miRNA. This intermediate miRNA is exported from the nucleus to the cytoplasm via Exportin-5 where the RNase III endonuclease Dicer generates the final mature miRNA. This short RNA loses one of its strands (the complementary miRNA* strand) while the other is loaded onto an Argonaute-containing RNA-induced silencing complex (RISC) which mediates gene silencing. Once the miRNA binds to its target gene, regulation takes place mainly through mRNA degradation or translation inhibition $[22,23]$ (see Figure 1). For simplicity, the widely used term "miRNA 
biogenesis" hereafter refers to the initial step of miRNA excision from its RNA transcript.

miRNAs can be located inter- or intragenically. When intergenic, their expression is coordinated with other miRNAs as a cluster $[25,26]$. When intragenic, namely, positioned within a protein-coding gene (almost exclusively in introns), they are often expressed from the same strand as their host-gene [27-30] and at correlated levels [31]. Given that both coding mRNAs and miRNAs are generated from the same transcriptional unit, and that they cooccur in close cellular proximity, it would be puzzling if these events exhibited total independence. Recent studies, from transcription, miRNA-processing, and splicing oriented perspectives, have investigated these fascinating interactions and yielded interesting, yet somewhat controversial findings.

\section{Intronic miRNA Biogenesis in Light of Pre-mRNA Splicing}

Relationships between intronic miRNAs and the processing events of their host mRNA, namely, transcription and splicing have been addressed. Here we outline the major studies in the field.

Expressed Sequence Tag (EST) libraries of expressed mRNAs are derived from various cells and tissues. The ESTs represent a snapshot of cellular transcripts at a particular time point and thus display the given mRNA plethora and its variety at a particular cellular state. Analysis of this data revealed several chimeric transcripts containing miRNA and part of the adjacent mRNA sequences [32]. At an early stage of miRNA research this indicated the existence of a shared RNA transcript. Notably, at a later stage, some of these EST fragments were shown to be partially spliced, with either $5^{\prime}$ or $3^{\prime}$ ends matching putative Drosha cleavage sites [30]. These results strengthened the possibility that miRNAs and mRNAs are processed from the same RNA substrate. In addition, the correlated expression pattern of host-gene transcripts and their miRNAs [31] suggested that miRNAs have coevolved to use the same promoter for transcription [26]. Along with this work, by comparing miRNA processing in a construct containing only the intronic sequence versus one that also includes the flanking exons, Pawlicki and Steitz [33] found that the levels of pri-miRNA transcribed from introns are increased in the presence of flanking exons, due to prolonged retention at the site of transcription. This supported the notion that flanking exons may facilitate miRNA processing by increasing the time pri-miRNAs spend tethered to the DNA template $[33,34]$. Altogether, the data indicates that intronic miRNA processing is enhanced by physical proximity to the site of transcription, and possibly also by splicing of the host gene. Several groups have isolated and identified various proteins associated with the human Microprocessor complex [35-37]. In these studies, numerous Microprocessor-associated proteins were identified as splicing factors (e.g., hnRNPH1; [37]) or involved in pre-mRNA processing (e.g., DHX15; [38]).

Taken together, based on miRNA-mRNA transcriptional (EST) evidence; shared promoters; facilitated biogenesis when flanked by exons; and overlapping proteins between the functional complexes, the data suggests that the Microprocessor is potentially enhanced and present during transcription and most likely also during splicing. If the same RNA substrate is subjected to both host-gene and intronic miRNA maturation, the intriguing question raised is how do all these processes-transcription, pre-mRNA splicing, and miRNA processing — crosstalk?

The complexity of the miRNA-host-gene interaction model has increased recently when studies from the Proudfoot Laboratory demonstrated that pre-miRNAs are generated through cotranscriptional cleavage by Drosha. Morlando et al. [39] suggested that efficient clearance of intronic sequences following Microprocessor (Drosha) cleavage may act to enhance the splicing efficiency. This occurs both in intergenic miRNAs and intronic miRNA genes [39]. These researchers showed that the Microprocessor complex, as well as $5^{\prime}-3^{\prime}$ and $3^{\prime}-5^{\prime}$ RNA exonucleases, are recruited to chromatin associated with intronic miRNAs during transcription of the host primary transcript, and that Drosha cleavage occurs before host intron splicing. They found that miRNA-harboring transcripts preferentially associated with chromatin fractions, from which they concluded that both pre-miRNA cleavage and intronic splicing must occur on the same nascent transcripts. The rapid exonucleolytic removal of intronic sequences may clear the proximal vicinity of RNA processing for the purpose of efficiently completing the premRNA splicing task. The enhancement of splicing by the Microprocessor does not agree with other studies [30, 40] (discussed below) and does not concur with experiments in yeast that showed enhanced processing for siRNA flanked exons in splicing mutants [41].

A crosstalk between two physically overlapping RNA transcripts is not unheard of. Dependencies are seen, for example, during the biogenesis process of small nucleolar RNA (snoRNA) [42-44]. In the process of snoRNA maturation, functional links between intronic snoRNP assembly, pre-mRNA synthesis and processing have been described [45-47]. An antithesis to this dependency is the alternative miRNA biogenesis pathway that bypasses the Microprocessor via generation of "Mirtrons" [48, 49]. This mechanistically distinct class of intronic miRNAs stem from very short introns where splicing substitutes the first step of miRNA biogenesis. In this case, splicing activities replace the requirements for a Microprocessor.

However, not all roads lead to the observed dependency between microprocessing and splicing. Ying and Lin [50] have designed an artificial intron containing a pre-miRNA secondary structure. They used this construct to show that the mature miRNA was released only from the spliced intron. This suggested that spliced introns are subsequently used by Drosha and argued against any physical link between the Microprocessor and the transcriptional unit or between the Microprocessor and the Spliceosome [50]. Dye et al. [40] showed that exons of pre-mRNA are tethered to the elongating RNA polymerase II either directly or indirectly without affecting processing, indicating that cotranscriptional cleavage of nascent intronic miRNA transcripts does not affect splicing efficiency $[40,51]$. Supporting the same 


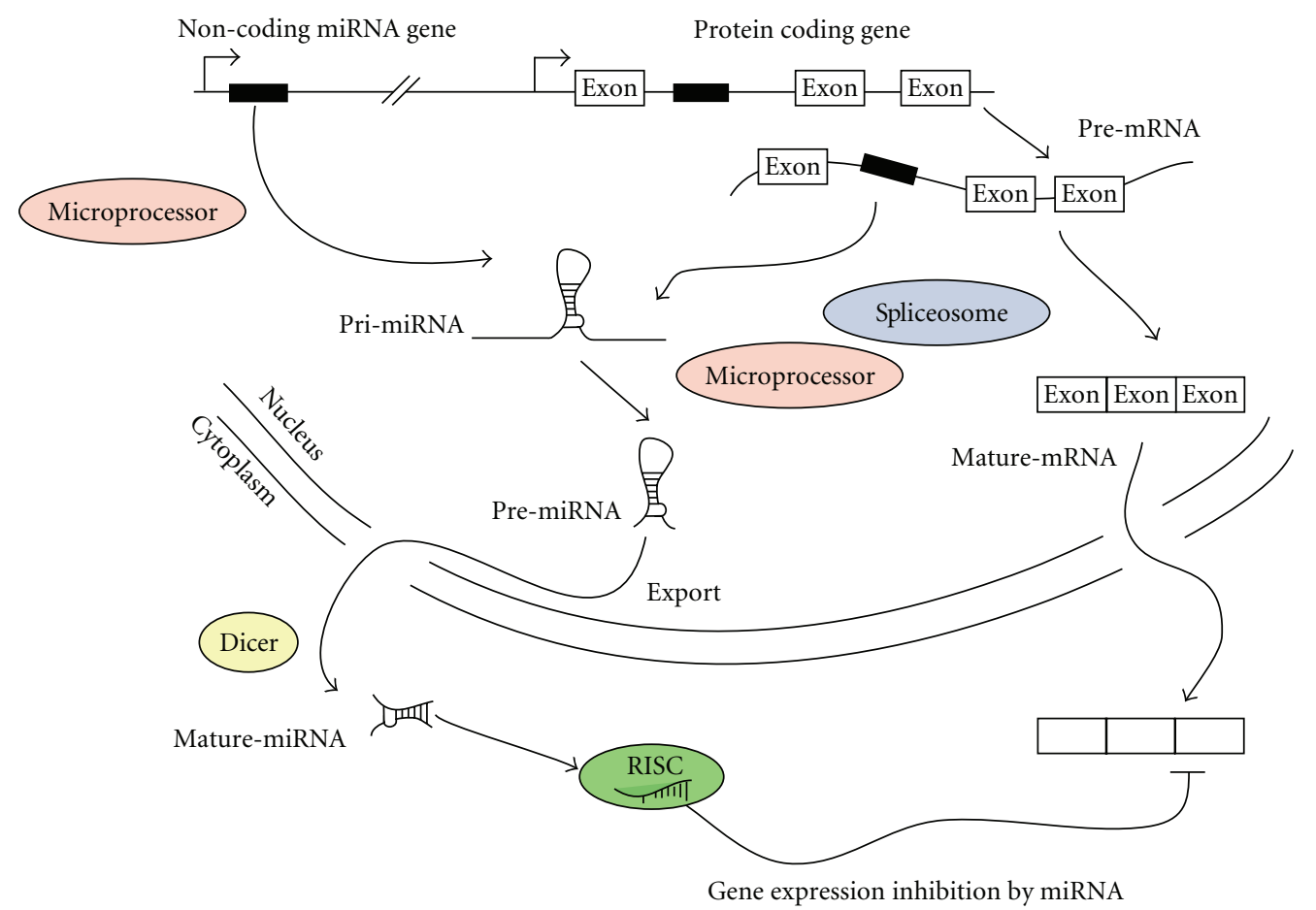

FIGURE 1: The canonical miRNA biogenesis pathway and its affect on gene expression. Elaborated mechanisms and exceptions to this pathway are reviewed in [24].

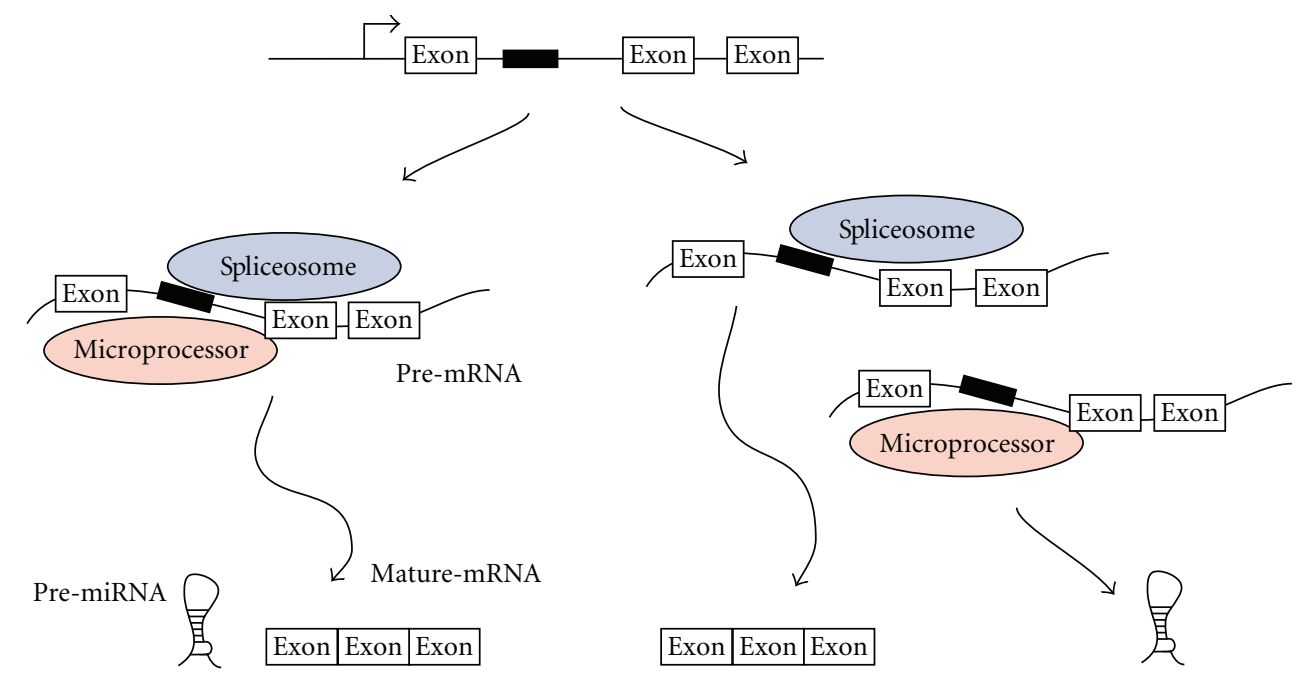

Figure 2: Possible models for intronic miRNA biogenesis. Either both miRNA and mRNA are generated from one RNA transcript (left scheme), or each is generated from an individual transcript (right scheme).

view, Kim and Kim [30] addressed miRNA biogenesis in light of the splicing mechanism. They demonstrated that cleavage of an intronic miRNA did not significantly affect the production of mature mRNA and, conversely, the production of mature miRNA was not significantly affected by splicing. In their experiments, knockdown of Drosha, or mutations in the miRNA hairpin, eliminated miRNA generation without dramatically affecting mRNA splicing. This suggested that miRNA biogenesis and splicing are coordinated but not functionally linked or interdependent. Taking a closer look, however, they also mention that Drosha knockdown led to a modest increase in spliced mRNA production and so did mutations in the miRNA hairpin. Their work showed that the adjacent introns were spliced more rapidly than miRNA-encoding introns, suggesting that binding of the Microprocessor may eventually interfere with the splicing to some extent. Taken together, Kim and Kim's data [30] imply mostly independent activities but cannot exclude the possibility that the Microprocessor interferes, to some level, with splicing. 


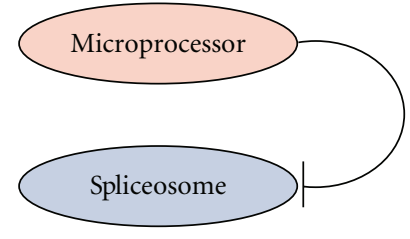

(a)

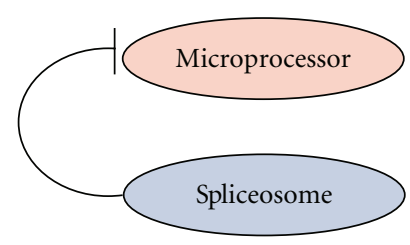

(b)

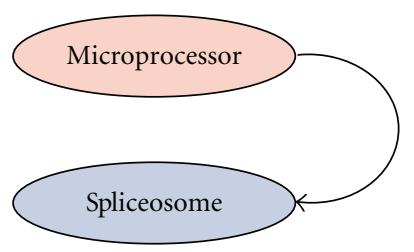

(c)

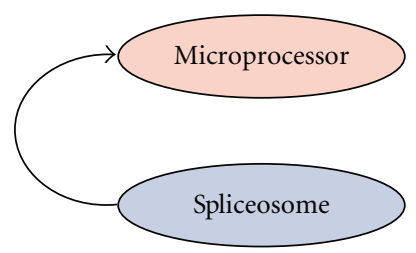

(d)
Figure 3: Possible crosstalk models between the miRNA biogenesis process, carried out by the Microprocessor, and the pre-mRNA splicing reaction, carried out by the Spliceosome.

Attempting to explain how both miRNA and mRNA are generated from the same DNA locus, reconciling with the studies described here, we come up with two distinct models. The first envisions a single miRNA and mRNA arising from the same RNA precursor. The second predicts generation of an miRNA and mRNA products arising from two independent RNA transcripts (see Figure 2). If the latter scenario was true, microprocessing and splicing would be independent of each other. The outcome would be either no functional hindrance between the Microprocessor and Spliceosome activities or a competition for available premRNA substrates. In the event that both RNA products originate from the same precursor, it is conceivable that the two processes happen consecutively. This would imply that execution of one process would be a prerequisite for the other to occur. Alternatively, miRNA processing and mRNA splicing may be coordinated so that the Microprocessor and Spliceosome interact with each other. This interaction may be minimal, without affecting the amounts of miRNA and mRNA produced-as was suggested recently [30], or it may constitute a level of regulation. We note that these events should always be looked at in the spatial-temporal context meaning that the Microprocessor might act at an independent rate prior to the Spliceosome assembly, and thus their direct interaction would be prevented. Due to accumulating evidence, both in favor and against dependencies between splicing and microprocessing, further investigation is necessary in order to uncover the full complexity of these processes.

\section{Coordinated Crosstalk}

Cellular regulatory events are commonly embedded in complex networks of interactions. The potential coor- dinated activity between the Microprocessor and the Spliceosome can be stratified into four possible relationships. The Microprocessor can inhibit or activate the Spliceosome, and the Spliceosome can inhibit or activate the Microprocessor (Figure 3). These relationships are not mutually exclusive. For example, the Microprocessor could activate the Spliceosome by recruiting splicing factors to intronic miRNAs, while at the same time the Spliceosome could inhibit microprocessing. We cannot rule out, however, that these relationships occur in one large complex depending on the presence of particular RNA processing proteins within the Microprocessor [37]. Many complex regulatory loops, both positive and negative, were seen in other cellular systems (e.g., see [52, $53])$.

The Spliceosome is a mega complex of hundreds of proteins and snRNPs [54]. Its assembly and dynamic structure have been extensively studied $[55,56]$. The Microprocessor, on the other hand, is composed of a handful of proteins $[21,36]$, minimally described as a two-protein complex $[24,35]$ (alternatively, see [37]). It is hard to visualize these two very differently-sized complexes aligned at the same position, competing for the same substrate. Thus, a coordinated processing and crosstalk seems necessary for these complexes-the Microprocessor and the Spliceosome-to be able to process the same transcript with intricate accuracy.

During the mRNA splicing process, the rate at which transcription takes place may affect the transcripts' pattern of splicing [57]. Thus, kinetics of intron removal and exon ligation may play a role in selecting particular spliced isoforms. Given that some introns undergo miRNA excision, unless the removal is extremely rapid, one can imagine a possible effect on splicing outcome. Thus, from an evolutionary perspective, an intronic miRNA might evolve to participate in determining splicing kinetics. Consequently, an evolutionary driving force may direct miRNA positioning within the intron to prevent disruption of relevant splice signals (also see [39]).

In summary, growing evidence indicates a complex crosstalk between transcription and splicing. It is not surprising then that microprocessing is also linked to these events. Many questions still remained unanswered. Are these processes coordinated by cis regulatory sequences or trans acting factors? Does one process dominate over the other? How widespread is the mechanism and does it govern all intronic miRNAs? To date, not all Microprocessor and Spliceosome crosstalk scenarios (as described in Figure 3) have been identified. Yet, given the complexity of cellular pathways, it is probably only a matter of time before their elucidation.

\section{Acknowledgments}

The authors would like to thank Kathleen Robinson, Adrienne Yanez, Steffen Schubert, Eyal Mor, Shira Modai, Naama Hazan, Yakir Vaknin, and Carl Novina for commenting on the manuscript. 


\section{References}

[1] T. Maniatis and R. Reed, "An extensive network of coupling among gene expression machines," Nature, vol. 416, no. 6880, pp. 499-506, 2002.

[2] T. W. Nilsen, "The spliceosome: the most complex macromolecular machine in the cell?" BioEssays, vol. 25, no. 12, pp. 1147-1149, 2003.

[3] M. J. Moore and N. J. Proudfoot, "Pre-mRNA processing reaches back to transcription and ahead to translation," Cell, vol. 136, no. 4, pp. 688-700, 2009.

[4] E. Kim, A. Goren, and G. Ast, "Alternative splicing: current perspectives," BioEssays, vol. 30, no. 1, pp. 38-47, 2008.

[5] E. T. Wang, R. Sandberg, S. Luo, et al., "Alternative isoform regulation in human tissue transcriptomes," Nature, vol. 456, no. 7221, pp. 470-476, 2008.

[6] E. C. Lai, "Micro RNAs are complementary to 3' UTR sequence motifs that mediate negative post-transcriptional regulation," Nature Genetics, vol. 30, no. 4, pp. 363-364, 2002.

[7] D. P. Bartel, "MicroRNAs: target recognition and regulatory functions," Cell, vol. 136, no. 2, pp. 215-233, 2009.

[8] S. Griffiths-Jones, H. K. Saini, S. van Dongen, and A. J. Enright, "miRBase: tools for microRNA genomics," Nucleic Acids Research, vol. 36, database issue, pp. D154-D158, 2008.

[9] B. P. Lewis, I.-H. Shih, M. W. Jones-Rhoades, D. P. Bartel, and C. B. Burge, "Prediction of mammalian microRNA targets," Cell, vol. 115, no. 7, pp. 787-798, 2003.

[10] A. Krek, D. Grun, M. N. Poy, et al., "Combinatorial microRNA target predictions," Nature Genetics, vol. 37, no. 5, pp. 495500, 2005.

[11] C. B. Nielsen, N. Shomron, R. Sandberg, E. Hornstein, J. Kitzman, and C. B. Burge, "Determinants of targeting by endogenous and exogenous microRNAs and siRNAs," RNA, vol. 13, no. 11, pp. 1894-1910, 2007.

[12] A. Grimson, K. K.-H. Farh, W. K. Johnston, P. Garrett-Engele, L. P. Lim, and D. P. Bartel, "MicroRNA targeting specificity in mammals: determinants beyond seed pairing," Molecular Cell, vol. 27, no. 1, pp. 91-105, 2007.

[13] J. Brennecke, A. Stark, R. B. Russell, and S. M. Cohen, "Principles of microRNA-target recognition," PLoS Biology, vol. 3, no. 3, p. e85, 2005.

[14] R. C. Friedman, K. K.-H. Farh, C. B. Burge, and D. P. Bartel, "Most mammalian mRNAs are conserved targets of microRNAs," Genome Research, vol. 19, no. 1, pp. 92-105, 2009.

[15] R. W. Carthew, "Gene regulation by microRNAs," Current Opinion in Genetics and Development, vol. 16, no. 2, pp. 203 208, 2006.

[16] R. Garzon, M. Fabbri, A. Cimmino, G. A. Calin, and C. M. Croce, "MicroRNA expression and function in cancer," Trends in Molecular Medicine, vol. 12, no. 12, pp. 580-587, 2006.

[17] R. Schickel, B. Boyerinas, S.-M. Park, and M. E. Peter, "MicroRNAs: key players in the immune system, differentiation, tumorigenesis and cell death," Oncogene, vol. 27, no. 45, pp. 5959-5974, 2008.

[18] V. Ambros, "The functions of animal microRNAs," Nature, vol. 431, no. 7006, pp. 350-355, 2004.

[19] L. P. Lim, N. C. Lau, P. Garrett-Engele, et al., "Microarray analysis shows that some microRNAs downregulate large numbers of-target mRNAs," Nature, vol. 433, no. 7027, pp. 769-773, 2005.

[20] W. Filipowicz, S. N. Bhattacharyya, and N. Sonenberg, "Mechanisms of post-transcriptional regulation by microRNAs: are the answers in sight?" Nature Reviews Genetics, vol. 9, no. 2, pp. 102-114, 2008.

[21] H. Seitz and P. D. Zamore, "Rethinking the microprocessor," Cell, vol. 125, no. 5, pp. 827-829, 2006.

[22] N. Bushati and S. M. Cohen, "MicroRNA functions," Annual Review of Cell and Developmental Biology, vol. 23, pp. 175-205, 2007.

[23] N. Shomron, D. Golan, and E. Horstein, "An evolutionary perspective of animal microRNAs and their targets," Journal of Biomedicine and Biotechnology. In press.

[24] J. Winter, S. Jung, S. Keller, R. I. Gregory, and S. Diederichs, "Many roads to maturity: microRNA biogenesis pathways and their regulation," Nature Cell Biology, vol. 11, no. 3, pp. 228$234,2009$.

[25] Y. Altuvia, P. Landgraf, G. Lithwick, et al., "Clustering and conservation patterns of human microRNAs," Nucleic Acids Research, vol. 33, no. 8, pp. 2697-2706, 2005.

[26] F. Ozsolak, L. L. Poling, Z. Wang, et al., "Chromatin structure analyses identify miRNA promoters," Genes and Development, vol. 22, no. 22, pp. 3172-3183, 2008.

[27] A. Rodriguez, S. Griffiths-Jones, J. L. Ashurst, and A. Bradley, "Identification of mammalian microRNA host genes and transcription units," Genome Research, vol. 14, no. 10A, pp. 1902-1910, 2004.

[28] N. Liu, K. Okamura, D. M. Tyler, M. D. Phillips, W.-J. Chung, and E. C. Lai, "The evolution and functional diversification of animal microRNA genes," Cell Research, vol. 18, no. 10, pp. 985-996, 2008.

[29] K. Okamura, W.-J. Chung, and E. C. Lai, “The long and short of inverted repeat genes in animals: microRNAs, mirtrons and hairpin RNAs," Cell Cycle, vol. 7, no. 18, pp. 2840-2845, 2008.

[30] Y.-K. Kim and V. N. Kim, "Processing of intronic microRNAs," EMBO Journal, vol. 26, no. 3, pp. 775-783, 2007.

[31] S. Baskerville and D. P. Bartel, "Microarray profiling of microRNAs reveals frequent coexpression with neighboring miRNAs and host genes," RNA, vol. 11, no. 3, pp. 241-247, 2005.

[32] N. R. Smalheiser, "EST analyses predict the existence of a population of chimeric microRNA precursor-mRNA transcripts expressed in normal human and mouse tissues," Genome Biology, vol. 4, no. 7, article 403, 2003.

[33] J. M. Pawlicki and J. A. Steitz, "Primary microRNA transcript retention at sites of transcription leads to enhanced microRNA production," Journal of Cell Biology, vol. 182, no. 1, pp. 61-76, 2008.

[34] J. M. Pawlicki and J. A. Steitz, "Subnuclear compartmentalization of transiently expressed polyadenylated pri-microRNAs: processing at transcription sites or accumulation in SC35 foci," Cell Cycle, vol. 8, no. 3, pp. 345-356, 2009.

[35] R. I. Gregory, K.-P. Yan, G. Amuthan, et al., "The Microprocessor complex mediates the genesis of microRNAs," Nature, vol. 432, no. 7014, pp. 235-240, 2004.

[36] R. I. Gregory, T. P. Chendrimada, and R. Shiekhattar, "MicroRNA biogenesis: isolation and characterization of the microprocessor complex," Methods in Molecular Biology, vol. 342, pp. 33-47, 2006.

[37] A. Shiohama, T. Sasaki, S. Noda, S. Minoshima, and N. Shimizu, "Nucleolar localization of DGCR8 and identification of eleven DGCR8-associated proteins," Experimental Cell Research, vol. 313, no. 20, pp. 4196-4207, 2007.

[38] X. Wen, S. Tannukit, and M. L. Paine, "TFIP11 interacts with mDEAH9, an RNA helicase involved in spliceosome 
disassembly," International Journal of Molecular Sciences, vol. 9, no. 11, pp. 2105-2113, 2008.

[39] M. Morlando, M. Ballarino, N. Gromak, F. Pagano, I. Bozzoni, and N. J. Proudfoot, "Primary microRNA transcripts are processed co-transcriptionally," Nature Structural and Molecular Biology, vol. 15, no. 9, pp. 902-909, 2008.

[40] M. J. Dye, N. Gromak, and N. J. Proudfoot, "Exon tethering in transcription by RNA polymerase II," Molecular Cell, vol. 21, no. 6, pp. 849-859, 2006.

[41] E. H. Bayne, M. Portoso, A. Kagansky, et al., "Splicing factors facilitate RNAi-directed silencing in fission yeast," Science, vol. 322, no. 5901, pp. 602-606, 2008.

[42] L.-H. Qu, Y. Henry, M. Nicoloso, et al., "U24, a novel intron-encoded small nucleolar RNA with two $12 \mathrm{nt}$ long, phylogenetically conserved complementarities to 28S rRNA," Nucleic Acids Research, vol. 23, no. 14, pp. 2669-2676, 1995.

[43] W. Filipowicz and V. Pogacic, "Biogenesis of small nucleolar ribonucleoproteins," Current Opinion in Cell Biology, vol. 14, no. 3, pp. 319-327, 2002.

[44] V. De Turris, G. Di Leva, S. Caldarola, F. Loreni, F. Amaldi, and I. Bozzoni, "TOP promoter elements control the relative ratio of intron-encoded snoRNA versus spliced mRNA biosynthesis," Journal of Molecular Biology, vol. 344, no. 2, pp. 383-394, 2004.

[45] T. Hirose, M.-D. Shu, and J. A. Steitz, "Splicing-dependent and -independent modes of assembly for intron-encoded box C/D snoRNPs in mammalian cells," Molecular Cell, vol. 12, no. 1, pp. 113-123, 2003.

[46] P. K. Yang, C. Hoareau, C. Froment, B. Monsarrat, Y. Henry, and G. Chanfreau, "Cotranscriptional recruitment of the pseudouridylsynthetase Cbf5p and of the RNA binding protein Naflp during H/ACA snoRNP assembly," Molecular and Cellular Biology, vol. 25, no. 8, pp. 3295-3304, 2005.

[47] P. Richard, A. M. Kiss, X. Darzacq, and T. Kiss, "Cotranscriptional recognition of human intronic box H/ACA snoRNAs occurs in a splicing-independent manner," Molecular and Cellular Biology, vol. 26, no. 7, pp. 2540-2549, 2006.

[48] E. Berezikov, W.-J. Chung, J. Willis, E. Cuppen, and E. C. Lai, "Mammalian mirtron genes," Molecular Cell, vol. 28, no. 2, pp. 328-336, 2007.

[49] J. G. Ruby, C. H. Jan, and D. P. Bartel, "Intronic microRNA precursors that bypass Drosha processing," Nature, vol. 448, no. 7149, pp. 83-86, 2007.

[50] S.-Y. Ying and S.-L. Lin, "Current perspectives in intronic micro RNAs (miRNAs)," Journal of Biomedical Science, vol. 13, no. 1, pp. 5-15, 2006.

[51] N. Gromak, G. Talotti, N. J. Proudfoot, and F. Pagani, "Modulating alternative splicing by cotranscriptional cleavage of nascent intronic RNA," RNA, vol. 14, no. 2, pp. 359-366, 2008.

[52] J. Y. Leung, F. T. Kolligs, R. Wu, et al., "Activation of AXIN2 expression by beta-catenin-T cell factor: a feedback repressor pathway regulating Wnt signaling," Journal of Biological Chemistry, vol. 277, no. 24, pp. 21657-21665, 2002.

[53] O. Brandman and T. Meyer, "Feedback loops shape cellular signals in space and time," Science, vol. 322, no. 5900, pp. 390 395, 2008.

[54] Z. Zhou, L. J. Licklider, S. P. Gygi, and R. Reed, "Comprehensive proteomic analysis of the human spliceosome," Nature, vol. 419, no. 6903, pp. 182-185, 2002.

[55] J. Sperling, M. Azubel, and R. Sperling, "Structure and function of the pre-mRNA splicing machine," Structure, vol. 16, no. 11, pp. 1605-1615, 2008.
[56] M. C. Wahl, C. L. Will, and R. Luhrmann, "The spliceosome: design principles of a dynamic RNP machine," Cell, vol. 136, no. 4, pp. 701-718, 2009.

[57] M. de la Mata, C. R. Alonso, S. Kadener, et al., "A slow RNA polymerase II affects alternative splicing in vivo," Molecular Cell, vol. 12, no. 2, pp. 525-532, 2003. 

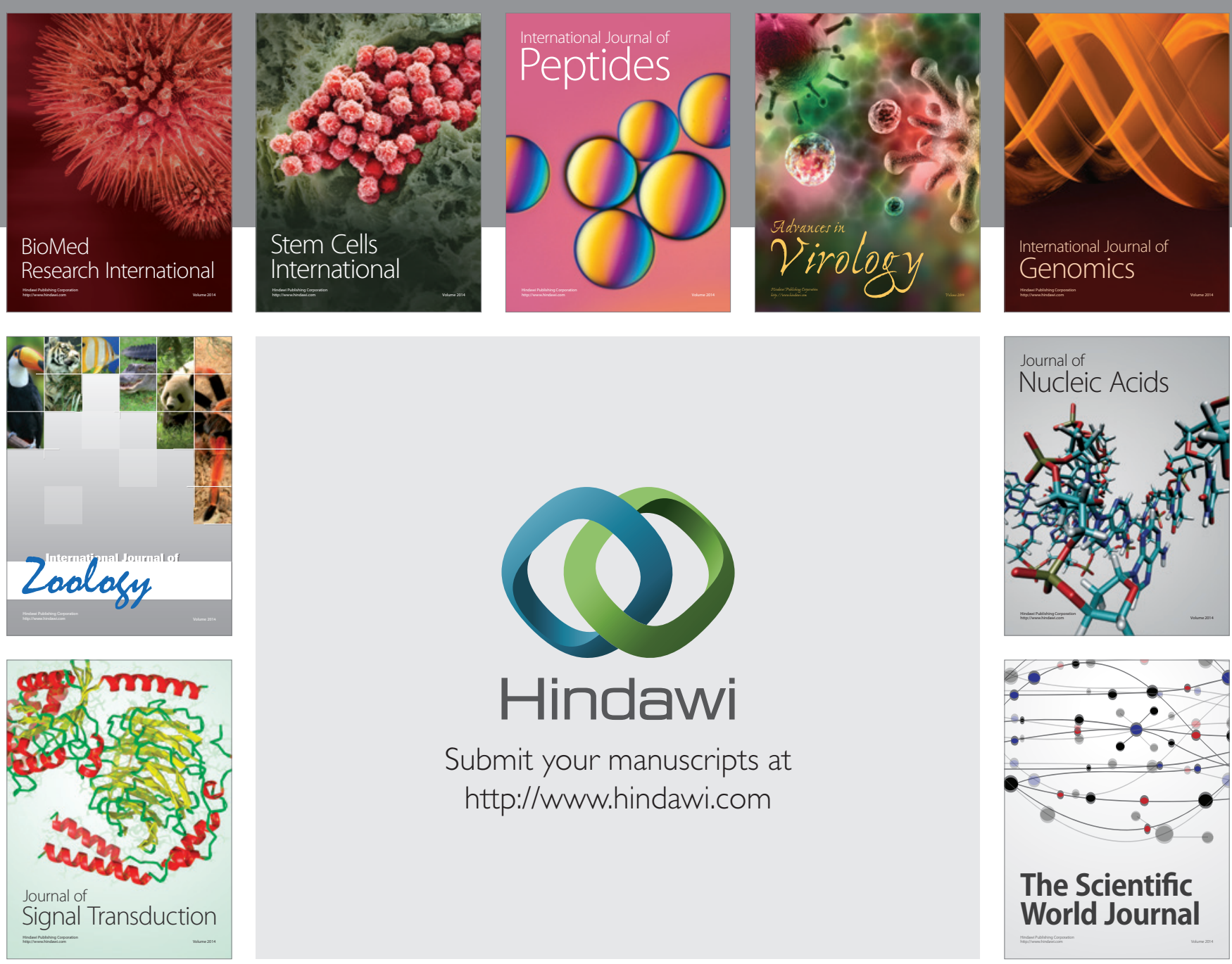

Submit your manuscripts at

http://www.hindawi.com
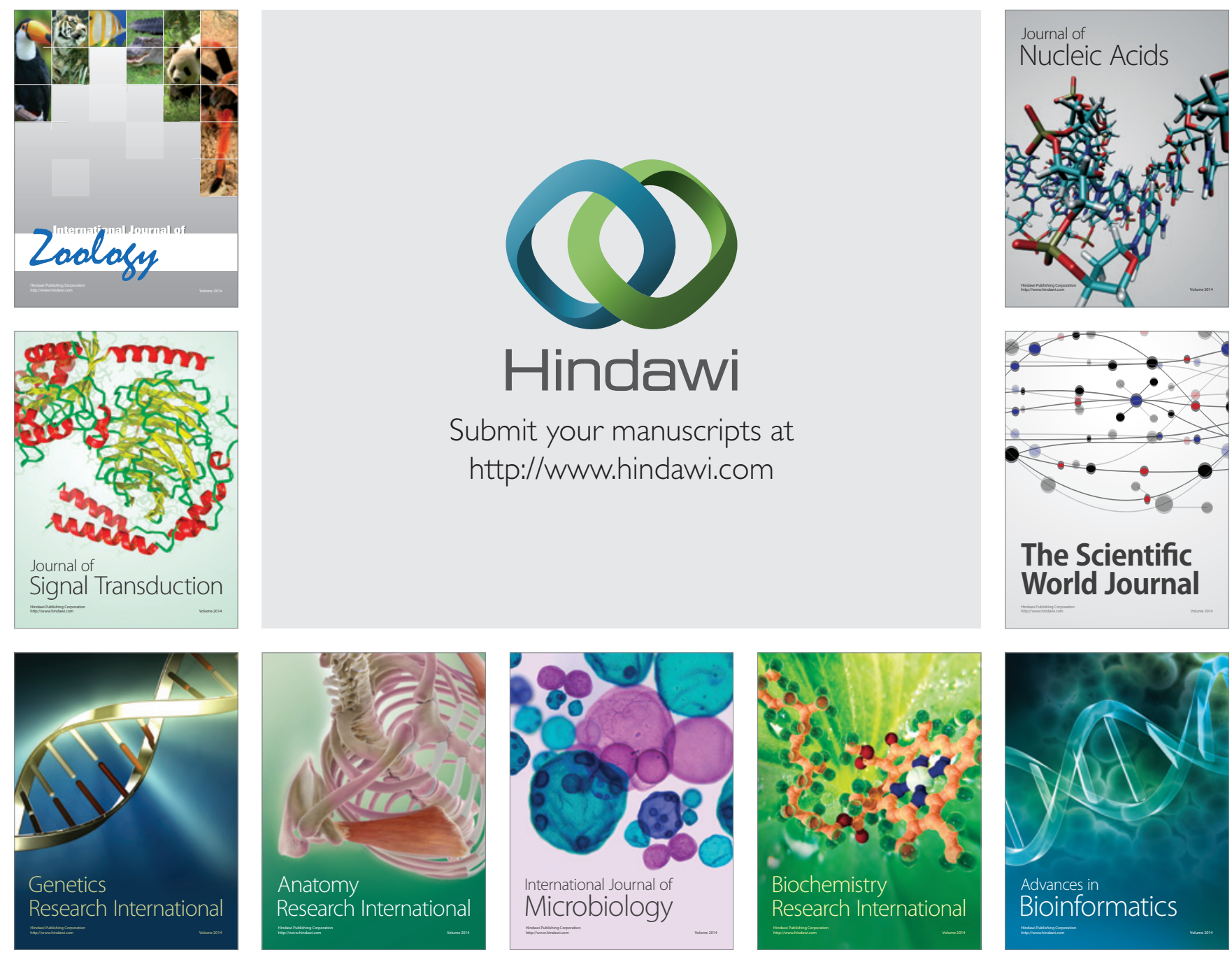

The Scientific World Journal
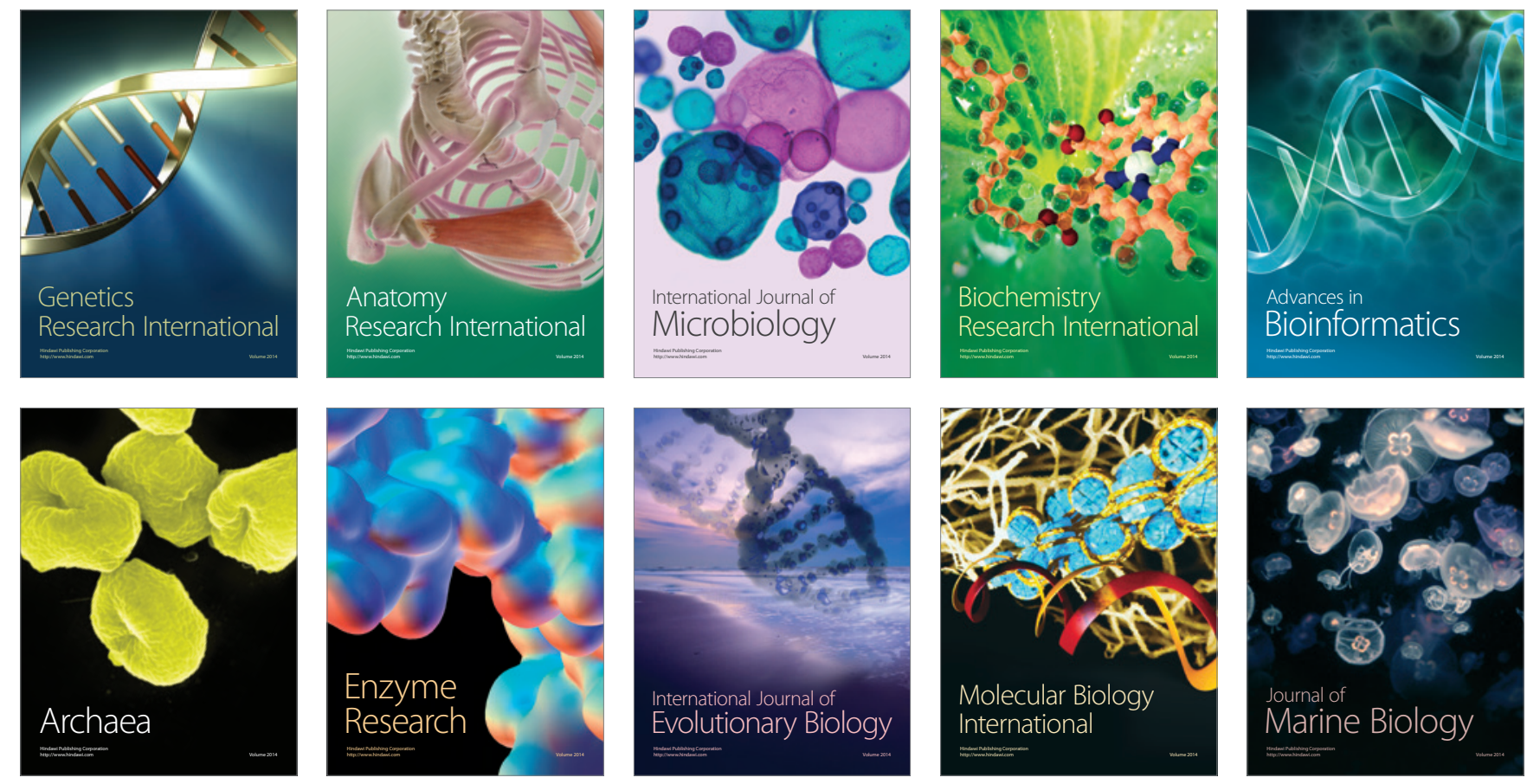